Wertmesser sind Züge der geistigen Kultur, repräsentiert durch Bildungsanstalten, Forschungs- und Kunstinstitutionen, sowie Wohlfahrtseinrichtungen. In dieser Hinsicht hat die Sowjetunion zweifellos mindestens ebenso beachtliche Anstrengungen gemacht wie in wirtschaftlicher, wenn sie auch keineswegs leicht beurteilt werden können. Dies mag abschließend noch die folgende Tabelle andeuten:

Schulen

Schüler

Lehrer

Hoch- und Fachschulen

Studierende

Wissenschaftliche Institute

Wissenschaftliche Mitarbeiter

Bibliotheken, Volks-

Kinos

Sanatorien und Krankenheime

Verfügbare Plätze

\begin{tabular}{rrrr}
$1914 / 15$ & $1927 / 28$ & $1940 / 41$ & $1955 / 56$ \\
\hline 124000 & 120000 & 199000 & 213000 \\
9656000 & 11589000 & 35528000 & 30070000 \\
280000 & 349000 & 1475000 & 1733000 \\
105 & 148 & 817 & 765 \\
127000 & 169000 & 812000 & 1867000 \\
. & 1263 & 1821 & 2950 \\
$\cdot$ & $\cdot$ & 98315 & 223893 \\
13880 &. & $95+00$ & 147200 \\
$1500 ?$ & 7300 & 28000 & 59300 \\
$\cdot$ &. & 1828 & 2178 \\
$(2000)$ & $(36100)$ & 239000 & $28+\infty 000$
\end{tabular}

Insgesamt läßt «Die UdSSR in Zahlen» erkennen, daß die Sowjetunion auf zahlreichen Bereichen des kulturellen Lebens unzweifelhafte Fortschritte gemacht hat und weiterhin zu erzielen gewillt ist. So manche Fragen das Werk noch offen läßt (insbesondere hinsichtlich der regionalen Struktur, die zwar, was die Unionsrepubliken betrifft, durch eine Reihe von Tabellen zur Darstellung gelangt, jedoch für geographische $Z$ wecke noch differenzierter wiedergegeben werden sollte), so wertvolle Erkenntnisse über das große Land bietet es uns doch im Ganzen und muß deshalb jedem Interessenten (und welcher Westeuropäer müßte dies nicht sein?) zum Studium empfohlen werden.

\title{
EIN INTERNATIONALER HOCHSCHULKURS FÜR KARTOGRAPHIE IN ZÜRICH UND BERN
}

\section{EDUARD IMHOF}

Die Eidg. Technische Hochschule in Zürich und die Eidg. Landestopographie in Wabern bei Bern führten vom 25. März bis 17. Mai 1957 erstmalig einen internationalen Hochschulkurs für Kartographie durch.

Veranlassung dazu war der aus zahlreichen Anfragen hervorgehende Wunsch ausländischer Fachkreise, die Fortschritte der theoretischen und praktischen Kartographie, wie sie in der Schweiz in neuerer Zeit erzielt worden waren, kennen zu lernen. Es handelte sich somit um einen kulturellen Beitrag der Schweiz an die übrige Welt, im Sinne der durch die UNESCO geförderten Bestrebungen.

Die wissenschaftliche und didaktische Leitung war Prof. Dr. h. c. Ed. Imhof, Vorsteher des Kartographischen Institutes der Eidg. Techn. Hochschule, anvertraut, die Organisation lag in den Händen von Prof. Dr. h. c. S. Bertschmans, des Direktors der Eidg. Landestopographie.

Lehrziel des Kurses war eine Vermittlung neuester methodischer Erkenntnisse und technischer Verfahren. Dies bedingte eine Ergänzung des theoretischen Unterrichtes durch praktische Arbeit und damit eine Beschränkung der Teilnehmerzahl auf 21 Personen.

Die Kursteilnehmer entstammten den verschiedensten europäischen Ländern und den Vereinigten Staaten von Amerika (USA). Es handelte sich hierbei zur Hauptsache um Kartographie-Fachleute in leitenden Stellungen, um Kartographie-Dozenten, Lehrer an Kartographenschulen usw. Ihre aktive Arbeit trug zum guten Gelingen des Kurses wesentlich bei.

In der Art der Durchführung nahm der Kurs eine Mittelstellung ein zwischen wissenschaftlichem Kongreß, akademischem Unterricht und Praktikantentätigkeit.

Die ersten vier Kurswochen wurden am Kartographischen Institut der Eidg. Techn. Hochschule in.Zürich durchgeführt. Hierbei wurde durch dessen Vorsteher, Prof. ImноF, in ca. 60 Vorlesungs- und Kolloquiumstunden eine moderne Kartographie- 
lehre eingehend entwickelt. Diese Lehre umfaßt sowohl die topographischen Karten (die allgemeinen oder Geländekarten), insbesondere deren Reliefdarstellung, wie auch die thematischen (angewandten oder speziellen) Karten. Durch zeichnerische Übungen (etwa 50 Stunden) wurde der Stoff weiter verarbeitet.

Den Zürcher Kurswochen folgten vier Wochen Praktikum an der Eidg. Landestopographie in Wabern bei Bern. Am Beispiel der Landeskarten der Schweiz wurde hier in Inhaltsgestaltung, Zeichnung und Reproduktionstechnik topographischer Karten eingeführt. Insbesondere wurden Situationsgravur auf schichtbedecktes Glas, Zeichnung und Gravur felsigen Gebietes und Karten-Schattierungstechniken geübt. Hierbei wirkten als Lehrkräfte die Herren BüHLER, UlMer und WITZlER und einige weitere Kartographen. Überdies wurden in Bern folgende Vorlesungen und Demonstrationen eingefügt: Dr. D. Chervet: Neueste Entwicklungen der kartographischen Reproduktionstechnik (6 Stunden); Prof. Dr. E. Imhof und Paul Bühler: Kartenbeschriftung (ca. 10 Stunden); Prof. Dr. S. Bertschmann: Die eidgenössichen Kartenwerke und Führung kartographischer Betriebe (4 Stunden).

Weitere Bereicherungen erfuhr das Kursprogramm durch Besichtigungen der Kartographischen Anstalten Art. Institut Orell Füßli in Zürich und Kümmerly छ Frey in Bern, der Karten- und Relief-Sammlungen der Eidg. Techn. Hochschule, der Zürcher Universität und des Helmhausmuseums in Zürich, wie auch des Alpinen Museums in Bern, ferner der mechanisch-optischen Werkstätten Heinrich Wild $A G$. in Heerbrugg, Kern छ Co. in Aarau und Haag-Streit in Bern. Überdies hatten diese drei Firmen zusammen mit den Instituten von Corradi in Zürich und Amsler in Schaffhausen während der Dauer des Kurses an der Eidg. Techn. Hochschule eine instruktive Ausstellung zeichnerisch-optischer Geräte und Hilfsmittel aufgebaut. Weitere Besichtigungen boten Gelegenheit, die plan- und kartentechnischen Arbeiten städtischer, kantonaler und eidgenössischer Amtsstellen kennen zu lernen.

Die Wochenendtage wurden von den meisten Kursteilnehmern reichlich zu Ausflügen in die verschiedensten Gegenden der Schweiz und in angrenzende Gebiete ausgenützt. Das stabile herrliche Frühlingswetter begünstigte solche Unternehmungen in hohem Maße.

Anläßlich einer Abschlußfeier am 16. Mai in Wabern bei Bern wurde einmütig der Wunsch nach Wiederholung solcher Kurse in Zürich und Bern und nach einem internationalen Zusammenschluß der Kartographie-Fachleute zum Ausdruck gebracht.

\section{DIE SAMMLUNG FÜR VÖLKERKUNDE DER UNIVERSITÄT ZÜRICH I $955 / 57$}

Mit 6 Abbildungen

DIE TÄTIGKEIT 1955/56

Im Berichtjahr fanden verschiedene Änderungen im Personalbestand der Sammlung statt: ab 1. Dezember 1955 ist Frl. Dr. E. Leuzinger, welche die Leitung des Rietbergmuseums übernahm, nur noch halbtägig als Konservatorin der Sammlung tätig. An ihre Stelle wurde die bis dahin als technische Hilfskraft tätige Frl. HeLen GuHL, ebenfalls mit nur halbtägiger Arbeitszeit als Konservatorin ernannt. Als weitere Hilfskräfte wurden von Mitte November bis Jahresende die Damen S. HaAs und R. Hứrlimann für die Ausarbeitung des Photo- und Diasarchivs angestellt. Außerdem stellten sich seit August Frau Dr. A. Garbade-Lachenal und seit Mitte Januar 1956 Frau Dr. E. ZiNGg während 2 Nachmittagen in der Woche für Arbeiten in der Sammlung freiwillig zur Verfügung, speziell für Katalogisierungsarbeiten in der Bibliothek und Beschriftung und Einordnung der Leica-Diapositive.

Nach dem Wegzug des geographischen Instituts, mit dem die Völkerkundesammlung früher mehrere Räume gemeinsam benützte, wurden ihr im Kollegiengebäude einige Zimmer zugewiesen, wovon eines für administrative $Z$ wecke bestimmt, ein weiteres als Bibliotheks- und Übungsraum des Seminars eingerichtet wurde, während ein hinter der Sammlung gelegener Saal zur Unterbringung der ILG-schen Abessinien-Sammlung ausgebaut werden soll und ein Nebenraum bereits als Magazinraum bezogen worden ist. Außerdem erhielt die Sammlung die Erlaubnis, ihren im Dachstock befindlichen Doublettenraum erheblich zu vergrößern. 